\title{
Oral Health Literacy Assessment: development of an oral health literacy instrument for Spanish speakers
} \author{
Leslie P. Zeldin, MSUP, MPH ${ }^{3}$ \\ 1 Pediatric Dentistry, UNC, Chapel Hill, NC, USA \\ 2 RAND Corporation, Los Angeles, CA, USA \\ 3 Health Policy and Management, University of North Carolina, Chapel Hill, NC, USA \\ 4 Department of Health Management and Policy, University of Michigan, Ann Arbor, MI, USA
}

Jessica Lee, DDS, MPH, PhD; Brian Stucky, PhD²; Gary Rozier, DDS, MPH³ Shoou-Yih Lee, PhD4;

\begin{abstract}
Keywords
oral health literacy; Spanish health literacy measurements; psychometrics.

\section{Correspondence}

Dr. Jessica Lee, Pediatric Dentistry, UNC, 228 Bruaer Hall, Chapel Hill, NC 27599. Tel.: 919-966-2739; Fax: 919-966-7992; e-mail: leej@dentistry.unc.edu. Jessica Lee is with Pediatric Dentistry, UNC. Brian Stucky is with RAND Corporation. Gary Rozier and Leslie P. Zeldin are with Health Policy and Management, University of North Carolina. Shoou-Yih Lee is with the Department of Health Management and Policy, University of Michigan.
\end{abstract}

Received: 11/24/2011; accepted: 10/5/2012.

doi: 10.1111/jphd.12000

Journal of Public Health Dentistry 73 (2013) 1-8

\begin{abstract}
Objective: To develop an oral health literacy instrument for Spanish-speaking adults, evaluate its psychometric properties, and determine its comparability to an English version.

Methods: The Oral Health Literacy Assessment in Spanish (OHLA-S) and English (OHLA-E) are designed with a word recognition section and a comprehension section using the multiple-choice format developed by an expert panel. Validation of OHLA-S and OHLA-E involved comparing the instrument with other health literacy instruments in a sample of 201 Spanish-speaking and 204 English-speaking subjects. Comparability between Spanish and English versions was assessed by testing for differential item functioning (DIF) using item response theory.

Results: We considered three OHLA-S scoring systems. Based on validity and reliability comparisons, 24 items were retained in the OHLA-S instrument. OHLA-S was correlated with another health literacy instrument, Spanish Test of Functional Health Literacy in Adults $(P<0.05)$. Significant correlations were also found between OHLA-S and years of schooling, oral health knowledge, overall health, and an understanding of written health-care materials $(P<0.05)$. OHLA-S displayed satisfactory reliability with a Cronbach Alpha of $0.70-0.80$. DIF results suggested that OHLA-S and OHLA-E scores were not comparable at a given level of oral health literacy.

Conclusions: OHLA-S has acceptable reliability and validity. OHLA-S and OHLA-E are two different measurement tools and should not be used to compare oral health literacy between English- and Spanish-speaking populations.
\end{abstract}

\section{Introduction}

In light of research evidence in medicine that highlights the importance of health literacy for patient knowledge and positive health behaviors and outcomes (1), health literacy has received increasing attention in oral health. Oral health literacy studies have been limited to English-speaking populations (2-4). The exclusion of Spanish-speaking people from oral health literacy studies is primarily because of the lack of appropriate measurement tools available in the Spanish language. Examining oral health and oral health literacy among Spanish speakers is essential because it may provide an

Disclosures: None of the authors has any financial interest related to the article. avenue to test potential interventions that may improve oral health outcomes. Until recently, they had been the fastest growing in the United States, and there is evidence of a high prevalence of dental disease in the Spanish-speaking population $(5,6)$.

Identifying individuals with inadequate oral health literacy is difficult because information regarding one's reading ability, comprehension, listening, and other necessary skills cannot be easily obtained. Educational attainment is often used as a proxy but is often limited and may overestimate an individual's oral health literacy level, as literacy is usually several grades lower than the attained educational level (7). To date, five instruments have been developed to measure oral health literacy in English speakers: a) Rapid Estimate of Adult Literacy in Dentistry-30 (8); b) Rapid Estimate of Adult 
Literacy in Dentistry-99 (9); c) Test of Functional Health Literacy in Dentistry (10); d) Oral Health Literacy Instrument (11); and e) Rapid Estimate of Adult Literacy in Medicine and Dentistry (12). Although Spanish language health literacy instruments have been developed in medicine, none have been develop for dentistry.

Our research team previously developed and validated the Rapid Estimate for Adult Literacy in Dentistry (REALD-30), a quick and easy-to-use oral health literacy instrument. The REALD-30 contains 30 items. It has been adopted in research and tested in various settings (2-4). The lack of a valid and reliable Spanish language oral health literacy instrument prevents the study of oral health literacy in Latinos and can bias inferences from population-based studies.

In this paper, we report our efforts to develop an instrument for Spanish speakers, named Oral Health Literacy Assessment in Spanish (OHLA-S), and test its comparability to an English language version of the same instrument, OHLA in English (OHLA-E). Both instruments use the same words in the REALD-30, but have an added dimension of a comprehension test. Similar to the development of REALD30, our intent is to develop a Spanish instrument that is short and easy to administer. The following sections describe the development of the OHLA-S, the methods employed to validate the instrument, the validation results, and the comparability of OHLA-S and OHLA-E.

\section{Methods}

\section{Instrument development}

The initial draft of OHLA-S contained the same 30 dental words as the REALD-30 (8). The REALD-30 is based on pronunciation of dental terms, and can assess an individual's oral health literacy because there is a strong association between pronunciation and comprehension among English speakers (10). REALD-30 scores range from 0 (lowest literacy) to 30 (highest literacy). Although a pronunciation instrument like REALD-30 may be an acceptable oral health literacy screening tool for English speakers, the phonetic structure of the Spanish language is highly regular - one sound is usually represented by one letter and vice versa (13). This feature of phonemegrapheme correspondence in the Spanish language violates the design basis of the REALD-30, making the test less appropriate for measuring health literacy in Spanish speakers. Thus, following the approach used by Lee and colleagues (14), we incorporated in the development of the OHLA multiplechoice items to assess the subject's comprehension (Table 1). Specifically, two common, simple words were chosen for each of the REALD-30 dental terms ("don't know" was also included as an option). One of the words, the key, was meaningfully associated with the REALD-30 dental term and the other, the distracter, was not. For example, for the REALD-30 word "sugar," the key word is "sweet" and the distracter word is "bitter." Because the purpose of the multiple-choice items was to verify the comprehension of the given dental terms, subjects were instructed not to guess. The reading difficulty of the key and distracter words was kept minimal to ensure that a person with a low literacy level could understand them.

An expert panel was formed to develop the OHLA instrument through a Delphi process (15). The panel consisted of nine members (six females and three males): three bilingual native Spanish-speaking dentists, three bilingual laypeople; and three who were not bilingual, but had extensive experience working with Spanish speakers in educational dental settings, and included two dentists and one sociologist who was also an expert in health literacy. To reflect our target population, the bilingual dentists and laypeople on the panel were of Mexican and Central American descent or had extensive experience working with these populations. All decisions made in the development of the OHLA instrument followed the same process where the facilitator provided the initial translation of English words into Spanish, taking into account both the dictionary definition and the commonality of usage in dental care and daily conversations, and then the panel met and through discussion either accepted or rejected the facilitator's initial translation. If the facilitator's decision was rejected, alternatives were provided and discussed until the group converged toward what was considered the "correct" translation.

The next step was to select the key and distracter words for each REALD word. An initial choice of key and distracter words was provided by the facilitator, followed by further suggestions by each panel member. All choices were combined into one document, and through a series of meetings and discussion the final terms were chosen by consensus of the expert panel. A pilot test was completed with five Englishspeaking and five Spanish-speaking subjects to help further refine the OHLA instrument.

\section{Data collection}

Three bilingual interviewers and one English-speaking interviewer conducted face-to-face interviews. Interviewers displayed the dental word and asked the respondent to pronounce it. If the word was pronounced correctly, the interviewer asked him or her to select the appropriately associated word. The interviewer recorded whether the respondent provided the correct verbal pronunciation (Yes or No) for each dental term as well as whether or not the respondent correctly associated the dental term to the key word if asked (i.e., of the two plausible choices, the one that matches in meaning the dental term, Table 1). "Don't know" was included as a response option to reduce guessing.

Subjects were recruited from eight sites that included:a) five Women's, Infants and Children's (WIC) Clinics from various geographic regions of the state; b) one Early Head Start center; 
Table 1 Oral Health Literacy Assessment in English and Spanish - OHLA-E and OHLA-S

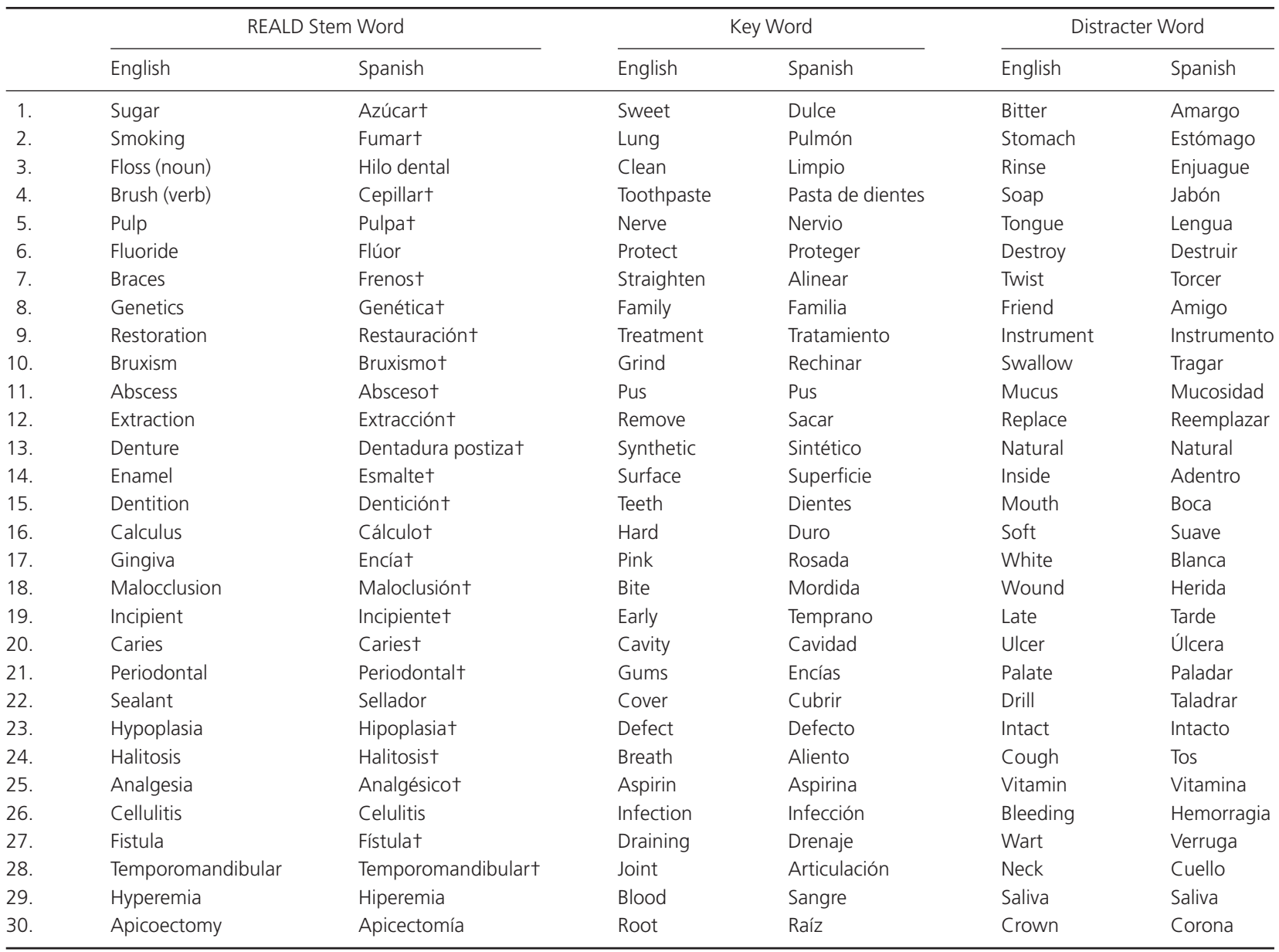

Note: $\uparrow$ indicates the 24 items appearing on the recommended OHLA-S.

c) one pediatric continuity care clinic at the University of North Carolina at Chapel Hill; and d) nearby private homes. To be eligible for participation in the study, the subjects had to meet the following criteria: a) be fluent in either English or Spanish; b) aged 18 or older but less than 80 years old; c) without obvious signs of cognitive impairment; d) without vision or hearing problems; and e) showing no sign of drug or alcohol intoxication. The entire interview took approximately 20 minutes and subjects were given a US $\$ 20$ gift card at the end of the interview. The research protocol was approved by the Biomedical Institutional Review Board at the University of North Carolina at Chapel Hill.

\section{Participant demographics}

The sample was comprised of 204 self-identified English speakers ( 85 percent female) and 201 self-identified Spanish speakers ( 82 percent female). The average age was 30.0 years (standard deviation $=8.3$ ). All Spanish-speaking participants were self-identified as of Latino or Hispanic origin,
93 percent were born outside of the United States ( 76 percent Mexico, 8 percent Honduras, 7 percent El Salvador, and 6 percent other), and 91 percent reported speaking primarily Spanish at home. For Spanish speakers, the average years of education was 9.0 ( standard deviation $=3.2$ ), corresponding to some high school education. The average years of education for English speakers was 13.0 (standard deviation = 2.2), corresponding to some education or training after high school. English speakers (52 percent) were more likely than Spanish speakers (31 percent) to have visited a dentist or dental clinic in the past year, $\chi^{2}(1)=17.9, P<0.01$.

\section{Scoring for OHLA-S}

Scoring for OHLA-S followed a previously tested system (16). This scoring method included both pronunciation and comprehension. Each OHLA-S item was assigned a score of 1 when the results of pronunciation and association tests were both correct. If either of the results were incorrect, the score for that particular item was 0 . In order to further examine 
the performance of OHLA-S, we considered the utility of two additional scoring methods. Each scoring method varied slightly in how "points" were awarded to the results of the pronunciation and association (comprehension) sections. The second of the three methods, extra credit for comprehension, assigned scores on a three-point scale: $0=$ incorrect pronunciation and incorrect association, $1=$ correct pronunciation and incorrect association, and $2=$ correct pronunciation and correct association. In other words, the second scoring method considered the association section to be extra and, if correct, worthy of an extra credit point. The third method, pronunciation only, excluding the results of the association section and considered only the correctness of pronunciation. This method of scoring is like other English word recognition tests such as Rapid Estimate of Adult Literacy in Medicine (REALM). We expected that the third approach would yield the least accurate assessment among the three methods for the OHLA-S because of the phoneme-grapheme correspondence in the Spanish language. Because OHLA-S is primarily a word recognition test with a comprehension component, no points were assigned if a subject was unable to correctly pronounce the dental term. All three methods considered the "don't know" category as an incorrect response.

\section{Psychometric evaluation of OHLA-S}

For each scoring method, a final scale was determined by selecting the subset of items that maximized score reliability using Cronbach's Alpha. The iterative process for determining the maximum reliability begins by considering all items (Table 1), and then sets aside the item that results in the greatest gain in score reliability. The algorithm continues until no additional gain in reliability can be made by setting aside items.

Next, a series of validity assessments was conducted to compare the results of the three OHLA-S scoring systems. Because the English version of OHLA was newly developed to examine comparability, it was also tested for validity. First, we assessed convergent validity by determining the Pearson correlation between OHLA-E and scores using each of the three OHLA-S scoring methods and the Test of Functional Health Literacy in Adults or TOFHLA in both Spanish and English (16). The Spanish TOFHLA has a reported reliability of alpha $=0.98$ (17). Past research has indicated strong associations for English-speaking samples between the TOFHLA and REALD-99 (9) and a two-stage REALD (18), suggesting the appropriateness of TOFHLA for testing convergent validity for the dental word recognition test.

We evaluated the predictive validity of the OHLA-S and OHLA-E by relating scores to four variables: a) years of education; b) self-reported general health according to a five-point Likert scale: poor, fair, good, very good, and excellent; c) oral health knowledge, measured as a count of correct answers to five questions ("The presence of gum disease can cause loss of a tooth," "The presence of gum disease can increase the risk of heart disease," "Fluoride disinfects water and makes it safe to drink," "Fluoride helps prevent tooth decay," and "Tooth decay can cause infections that spread to the face and other parts of the body"); and d) oral health-related quality of life, assessed using the Oral Health Impact Profile (OHIP-14) (19). The English language OHIP was translated into Spanish for use in this study based on two publications $(20,21)$. The resulting scale "Spanish OHIP" scale had an Alpha $=0.95$ in the current study sample. We hypothesized that high oral health literacy would be positively associated with high educational attainment, good general health, high oral health knowledge, and good oral health-related quality of life.

Additionally, OHLA-S scores were used to predict subjects' self-reported difficulties understanding written medical and dental materials and their perceived self-efficacy in completing medical forms using questions based on those developed by Chew and colleagues (22): "How often do you have someone help you read materials you might receive from a hospital or clinic?" "How often do you have problems understanding written information about the health of your teeth, mouth or jaws?" and "How confident are you filling out medical forms by yourself?" Response categories for the two frequency items were scored according to a four-point Likert scale: Always, Sometimes, Rarely, and Never. Responses for the confidence item were one of three responses: "Not at all confident," "Somewhat confident," or "Extremely confident" (high scores indicate high levels of self-efficacy). For ease of interpretation and analysis, we developed a summary scale derived from the sum of Likert scale scores for the items assessing self-reported difficulties understanding written materials and perceived self-efficacy in completing forms items. All three scoring methods for OHLA-S were compared to this scale (Table 3 ).

\section{Comparisons of OHLA-S with OHLA-E}

Another aim of the present study was to determine the degree to which Spanish and English versions of the OHLA produced equivalent scores. Researchers commonly compare scores on literacy scales across languages. Such comparisons implicitly assume that the scales measure the same underlying literacy level. However, one often finds for various types of measures that, after controlling for mean level group differences, items have different properties across subgroups (23). The English and Spanish versions of OHLA could be measuring literacy differently because of translation effects or phonetic differences in the two languages. This form of item bias often goes undetected when investigators consider only mean and variance differences across language. Thus, we evaluated equivalent OHLA scores between Spanish and 
Table 2 Correlations between Three Potential Scoring Methods and Various Assessments of Validity

\begin{tabular}{|c|c|c|c|c|c|c|}
\hline Score method (alpha) & TOFHLA & $\begin{array}{l}\text { Oral health } \\
\text { knowledge }\end{array}$ & OHIP & $\begin{array}{l}\text { Medical } \\
\text { materials }\end{array}$ & $\begin{array}{l}\text { Years } \\
\text { education }\end{array}$ & $\begin{array}{l}\text { Overall } \\
\text { health }\end{array}$ \\
\hline $\begin{array}{l}\text { OHLA-S pronunciation and } \\
\text { comprehension }(0.70)\end{array}$ & 0.53 & 0.26 & 0.29 & 0.28 & 0.44 & 0.18 \\
\hline $\begin{array}{l}\text { OHLA-S comprehension extra } \\
\text { credit }(0.78)\end{array}$ & 0.58 & 0.16 & 0.32 & 0.26 & 0.44 & 0.13 \\
\hline $\begin{array}{l}\text { OHLA-S pronunciation only } \\
(0.80)\end{array}$ & 0.56 & 0.02 & 0.35 & 0.20 & 0.34 & 0.06 \\
\hline $\begin{array}{l}\text { OHLA-E pronunciation and } \\
\text { comprehension }(0.82)\end{array}$ & 0.49 & 0.33 & 0.48 & 0.32 & 0.31 & 0.05 \\
\hline
\end{tabular}

Correlations in bold are $P \leq 0.05$.

Medical Materials, three items measuring patients' difficulties in understanding written medical and dental materials; OHIP, Oral Health Impact Profile; OHLA-E, Oral Health Literacy Assessment in English; OHLA-S, Oral Health Literacy Assessment in Spanish; TOFHLA, Test of Functional Health Literacy in Adults.

English speakers by testing for potential differential item functioning (DIF) using Likelihood Ratio (LR) tests in an item response theory (IRT) framework as implemented in IRTLRDIF (24). The test for DIF takes into account any mean or variance differences in the English- and Spanish-speaking groups and examined if OHLA performs the same in the respective groups. If OHLA-S and OHLA-E are comparable then no DIF should be present.

\section{Results}

\section{OHLA-S reliability}

The procedures used to maximize reliability resulted in scales of varying length for the different scoring methods. The pronunciation and comprehension scoring method produced an instrument that consisted of 24 items and had a reliability of alpha $=0.70$. The comprehension extra credit method resulted in a 26 -item instrument that had a reliability of alpha $=0.78$. Finally, the pronunciation only method produced a scale containing 23 items with a reliability of alpha $=0.80$. While the three methods set aside some different items to maximize reliability, a few items were consistently removed because they lacked variability. Those items included "brush," which had few individuals providing incorrect responses, "sealant," which had few correct associations and "cellulitis," which had few incorrect pronunciations, but was difficult to correctly associate.

\section{OHLA-S validity}

Table 2 displays correlations for the OHLA-E and each of the three OHLA-S scoring methods and the six variables used to assess validity. We observe consistently statistically significant associations between the OHLA-S and the variable used to determine convergent validity (TOFHLA) and three of the variables used to determine criterion-related predictive validity (OHIP, understanding written health materials, and years of education). However, only the pronunciation and comprehension scoring method was significantly related $(P<0.05)$ to all variables used in validity assessments.

Three separate linear regression models were fit to the data such that each of the three potential OHLA-S scores (i.e., the pronunciation and comprehension, comprehension extra credit, and pronunciation only scoring methods) were predicted from the summary score of patients' understanding of medical health information after controlling for age, gender, and years of education (Table 3 ). All three final models accounted for a significant proportion of the variability in OHLA-S scores (21 percent of the pronunciation and comprehension and comprehension extra credit score variance, and 15 percent of the pronunciation only score variance). However, after controlling for all other covariates, the summary score of a patient's self-perceived need for help in understanding written medical material remained a significant predictor of only the pronunciation and comprehension scoring method $(\beta=0.22$, se $=0.10, P<0.05)$.

\section{OHLA-S and OHLA-E validity comparisons using DIF}

Because OHLA-E is very similar to the frequently used REALD (2-4) and the pronunciation and comprehension method had the best performance in the OHLA-S, we scored the OHLA-E using only this method to conduct the comparability tests. However, the assessment of equivalence of scores between language groups indicated a high level of item bias. Of the 24 items in the pronunciation and comprehension scoring method, 23 were tested for DIF (one item was set aside from the English group for lack of variability). Seventeen of these items had significant DIF (i.e., item bias), indicating that the English and Spanish speakers used a different response process in responding to items. The difference in 
Table 3 Separate Linear Regressions Predicting Three Potential OHLA-S Scoring Systems

\begin{tabular}{|c|c|c|c|c|c|c|c|c|c|c|c|c|}
\hline & \multicolumn{4}{|c|}{$\begin{array}{l}\text { OHLA-S pronunciation } \\
\text { and comprehension }\end{array}$} & \multicolumn{4}{|c|}{$\begin{array}{c}\text { OHLA-S comprehension } \\
\text { extra credit }\end{array}$} & \multicolumn{4}{|c|}{ OHLA-S pronunciation only } \\
\hline & $\beta$ & SE & $t$ value & $P$ value & $\beta$ & SE & $t$ value & $P$ value & $\beta$ & SE & $t$ value & $P$ value \\
\hline Gender* & 0.38 & 0.54 & 0.70 & 0.49 & 1.66 & 1.02 & 1.63 & 0.11 & 0.93 & 0.54 & 1.70 & 0.09 \\
\hline Age & 0.03 & 0.03 & 0.81 & 0.42 & 0.00 & 0.06 & -0.02 & 0.99 & -0.03 & 0.03 & -0.96 & 0.34 \\
\hline Educationt & 0.40 & 0.07 & 5.46 & $<0.01$ & 0.80 & 0.14 & 5.81 & $<0.01$ & 0.33 & 0.07 & 4.47 & $<0.01$ \\
\hline Medical materialsł & 0.22 & 0.10 & 2.21 & 0.03 & 0.32 & 0.19 & 1.71 & 0.09 & 0.12 & 0.10 & 1.20 & 0.23 \\
\hline
\end{tabular}

* Gender: Males = 0, Females = 1.

† Education: Years of schooling.

₹ Medical materials: three items measuring patients' difficulties in understanding written medical and dental materials.

OHLA-S, Oral Health Literacy Assessment in Spanish; SE, standard error.

validity took into account mean and variances differences between speakers (Table 4). Because OHLA-S and OHLA-E scores were not equivalent, we did not perform traditional between-group tests (e.g., $t$-tests). Such comparisons could be misleading. Alternatively, Table 4 provides separate mean and variance estimates for English and Spanish speakers for the 23 items. While the association aspect of the scale resulted in reasonably similar scores, the pronunciation aspect was clearly less difficult for Spanish speakers, suggesting a potential explanation for item bias across subgroups.

\section{Discussion}

To date, the Spanish-speaking population has been largely ignored in oral health literacy studies. This absence of studies is primarily because of the lack of adequate health literacy measurement tools for this population. This paper reports the development and validation of the OHLA-S, a tool designed to measure oral health literacy in Spanish-speaking populations. Our findings indicate that each OHLA-S scoring system has both good predictive and convergent validity, and results in acceptable reliability (alpha values ranging from 0.70 to 0.80 ). These validity results were similar to those found in testing the REALD-30 (8). The English version of OHLA performed well and could be used in settings where there would be additional benefit in capturing a comprehension component to health literacy. Because the results of

Table 4 OHLA-S and OHLA-E Average Percent Correct

\begin{tabular}{lll}
\hline Language & Association (SD) & Pronunciation (SD) \\
\hline OHLA-S & $60 \%(3.3)$ & $86 \%(2.7)$ \\
OHLA-E & $66 \%(3.2)$ & $62 \%(4.6)$ \\
\hline
\end{tabular}

Note: OHLA-E and OHLA-S percentages are out of a score of 23 (e.g., an English pronunciation average of 14.2 indicates a percentage correct of $62 \%$; a Spanish pronunciation average of 19.9 indicates a percentage correct of $86 \%$ ).

OHLA-E, Oral Health Literacy Assessment in English; OHLA-S, Oral Health Literacy Assessment in Spanish; SD, standard deviation.
OHLA-E were similar to REALD-30, it is still unknown what the added value of the comprehension component would be and should be an area of future investigations.

While each of the three scoring methods tested has its own unique merits, we recommend the pronunciation and comprehension scoring method confirming our hypothesis of the added value of the addition of a comprehension component for measuring oral health literacy in Spanish-speaking populations. The instrument resulting from this scoring method contains only 24 items, is easy to administer, takes only 2-3 minutes and require minimal training. We came to the conclusion that the pronunciation and comprehension method of scoring the OHLA-S should be recommended for a number of reasons. It resulted in the best balance between reliability and validity. This method conceptualized correct responses as containing both accurate pronunciation and correct association, similar to the approach used in a previous study that developed a Spanish language health literacy instrument $(14,16)$. While the other scoring systems (comprehension extra credit and pronunciation only) result in slight gains in reliability, we noted a trade-off in evidence of validity.

We also found that our recommended OHLA-S instrument had a higher correlation with years of schooling in both pronunciation and comprehension and comprehension extra credit scoring approaches than the pronunciation only method. Because the pronunciation and comprehension and comprehension extra credit scoring methods took into consideration comprehension, this result was not surprising. Spanish speakers with higher levels of education may be better able to not only pronounce a word but also have the ability to comprehend the dental terms.

Additionally, because of the regular phonetic structure of the language, Spanish speakers may be able to read and correctly pronounce a term without necessarily understanding the meaning of the term. Therefore, the Spanish OHLA is designed to assess an individual's reading as well as comprehension skills in the dental care context. Word recognition instruments are based on the assumption that reading ability is a basic literacy skill and those with higher reading ability 
have less difficulty functioning in and negotiating the healthcare system. Along these lines, the present study is the first to find an association between oral health literacy and Spanishspeaking individuals' self-reported ability to understand medical health information and materials (controlling for known covariates), which is a key component in effectively operating within the health-care system.

Because our results indicated the presence of DIF, we were unable to confirm equivalent scores for OHLA-E and OHLA-S. While the association aspect of the scale resulted in reasonably similar scores, the pronunciation aspect was clearly less difficult for Spanish speakers (Table 4). These results, surprisingly, differ from those reported in a previous study that used a similar design to develop comparable instruments for assessing the literacy level of English speakers and Spanish speakers in the medical care context (16). We hypothesize the reason for our failed attempt to develop comparable instruments of oral health literacy for English- and Spanish-speaking adults may be due to the differences between the two groups in their understanding of and experience with oral health and dental care. In particular, individuals growing up in Latin American culture may have limited access to dental care and be unfamiliar with oral health terms. However, we are unable to determine if the differences are in their understanding and perception of oral health or in their literacy in the present study. Additional research is needed to understand how Spanish-speaking populations seek and process oral health information and how they navigate the dental health-care system.

The results presented here should be considered in light of the study's limitations. The data were collected from a nonprobability convenience sample of mostly clients from five NC-WIC clinics ( -380 participants). Future research should draw from a more representative sample. Also, the majority of our Spanish-speaking population in this current study was from Mexico. Further examination of validity and reliability of OHLA-S may be needed in different Latino subpopulations.

As with other oral health literacy instruments, such as REALD (8,9), REALM-D (12), and OHLI (11), OHLA is largely a reading instrument. It is unlikely that one instrument, like OHLA, can adequately serve all the needs for health literacy assessments in dentistry. While a reading test can provide a quick and easy assessment for patient care settings, future research is needed to not only test and refine OHLA-S, but to develop an instrument that more comprehensively measures literacy in the dental context.

\section{Acknowledgments}

This research was supported by grants from the National Institute of Dental and Craniofacial Research (RO1DE018236 and RO1DE0180451).

\section{Disclaimers}

The content is solely the responsibility of the authors and does not necessarily represent the official views of the National Institute of Dental and Craniofacial Research, or the University of North Carolina at Chapel Hill.

\section{References}

1. Dewalt DA, Berkman ND, Sheridan S, Lohr KN, Pignone MP. Literacy and health outcomes: a systematic review of the literature. J Gen Intern Med. 2004;19:1228-39.

2. Miller EK, Lee JY, DeWalt DA, Vann WF Jr. Impact of caregiver health literacy on children's oral health outcomes. Pediatrics. 2010;126:107-14.

3. Vann WF Jr, Lee JY, Baker D, Divaris K. Oral health literacy among female caregivers: impact on the oral health outcomes in early childhood. J Dent Res. 2010;89(12):1395-400.

4. Parker E, Jamieson L. Associations between Indigenous Australian oral health literacy and self-reported oral health outcomes. BMC Oral Health. 2010;10:3. [cited 2011 Jan 10]. Available from: http://www.biomedcentral.com/ 1472-6831/10/3.

5. US Department of Health and Human Services. The initiative to eliminate racial and ethnic disparities in health. Eliminating Minority Health Disparities. [cited 2011 Jan 13]. Available from: http://minorityhealth.hhs.gov/NPA/Files/ Plans/HHS?HHS_plans.pdf.

6. Dye BA, Tan S, Smith V, Lewis BG, Barker LK, Thornton-Evans G et al. Trends in oral health status: United States, 1988-1994 and 1999-2004. National Center for Health Statistics. Vital Health Stat. 2007;11(248):201-51.

7. American Medical Association. Ad Hoc Committee on health literacy for the council on scientific affairs. JAMA. 1999;281(1):552-7.

8. Lee JY, Rozier RG, Lee SY, Bender D, Ruiz R. Development of a word recognition instrument to test health literacy in dentistry: the REALD-30. J Public Health Dent. 2007;67(2): 94-8.

9. Richman JA, Lee JY, Rozier RG, Gong D, Pahel BT, WFJr V. Evaluation of a word recognition instrument to test health literacy in dentistry: the REALD-99. J Public Health Dent. 2007;67(2):99-104.

10. Gong DA, Lee JY, Rozier RG, Pahel BT, Richman JA, Vann WF Jr. Development and testing of the Test of Functional Health Literacy in Dentistry (TOFHLiD). J Public Health Dent. 2007;67(2):105-12.

11. Sabbahi D, Lawrence HP, Limeback H, Rootman I. Development and evaluation of an oral health literacy instrument for adults. Community Dent Oral Epidemiol. 2009;37:451-62.

12. Atchison KA, Gironda MW, Messadi D, Der-Martirosian C. Screening for oral health literacy in an urban dental clinic. J Public Health Dent. 2010;70(10):269-75. 
13. Nurss JR, Baker DW, David TC, Parker RM, Williams MV. Difficulties in functional health literacy screening in Spanish-speaking adults. J Read. 1995; 38:632-7.

14. Lee SY, Bender DE, Ruiz RE, Cho YI. Development of an easy-to-use Spanish Health Literacy test. Health Serv Res. 2006;41 (4 Pt 1):1392-412.

15. Turoff M. The design of a policy Delphi. Technol Forecast Soc Change. 1970;2:149-71.

16. Lee SY, Stucky B, Lee JY, Rozier RG, Bender D. Short assessment of health literacy-Spanish and English: a comparable test of health literacy for Spanish and English-speakers. Health Serv Res. 2010;45:1105-20.

17. Parker RM, Baker DW, Williams MV, Nurss JR. The test of functional health literacy in adults: a new instrument for measuring patients' literacy skills. J Gen Intern Med. 1995;10(10):537-41.

18. Stucky B, Lee JY, Lee SY, Rozier RG. Application of multi-stage testing using the REALD-30: a dental health literacy word recognition instrument for dentistry. Community Dent Oral Epidemiol. 2011. doi: 10.1111/j.1600-0528.2011.00619.x.
19. Slade GD. Derivation and validation of a short-form oral health impact profile. Community Dent Oral Epidemiol. 1997;25:284-90.

20. Quant SA, Hiott AE, Grzywacz JG, David SW, Arcury TA. Oral health and quality of life in migrant and seasonal farm workers in North Carolina. J Agricul Safety and Health. 2007;13(1):45-55.

21. Montero-Martín J, Bravo-Pérez M, Albaladejo-Martínez A, Hernández-Martín L, Rosel-Gallardo EM. Validation the oral health impact profile (OHIP-14sp) for adults in Spain. Med Oral Patol Or Oral Cir Bucal. 2009;14(1):E44-50.

22. Chew LD, Bradley KA, Boyko EJ. Brief questions to identify patients with inadequate health literacy. Fam Med. 2004;36(8):588-94.

23. Thissen D, Steinberg L, Wainer H. Use of item response theory in the study of group differences in trace lines. In: Wainer H, Braun HI, editors. Test validity. Hillside, NJ: Erlbaum; 1988. p. 124-69.

24. Thissen D. IRTLRDIF v.2.0b: software for the computation of the statistics involved in item response theory likelihood-ratio tests for differential item functioning [computer software]. University of North Carolina at Chapel Hill; 2001. 